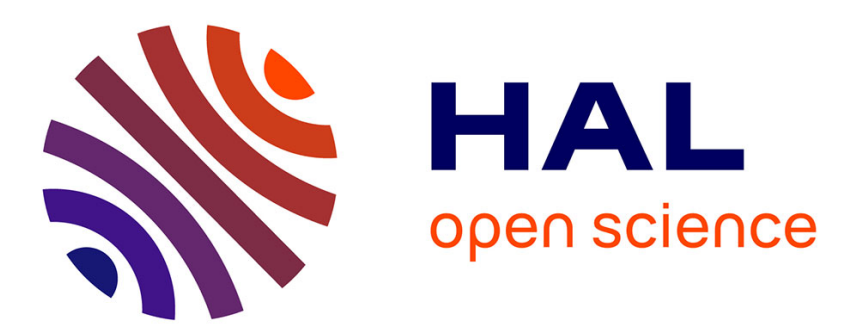

\title{
Indirect Tertiary Alcohol Enantiocontrol by Acylative Organocatalytic Kinetic Resolution
}

Titouan Desrues, Xueyang Liu, Jean-Marc Pons, Valérie Monnier, Jean-Arthur Amalian, Laurence Charles, Adrien Quintard, Cyril Bressy

\section{To cite this version:}

Titouan Desrues, Xueyang Liu, Jean-Marc Pons, Valérie Monnier, Jean-Arthur Amalian, et al.. Indirect Tertiary Alcohol Enantiocontrol by Acylative Organocatalytic Kinetic Resolution. Organic Letters, 2021, 23 (11), pp.4332 - 4336. 10.1021/acs.orglett.1c01261 . hal-03255245

\author{
HAL Id: hal-03255245 \\ https://hal.science/hal-03255245
}

Submitted on 14 Jun 2021

HAL is a multi-disciplinary open access archive for the deposit and dissemination of scientific research documents, whether they are published or not. The documents may come from teaching and research institutions in France or abroad, or from public or private research centers.
L'archive ouverte pluridisciplinaire $\mathbf{H A L}$, est destinée au dépôt et à la diffusion de documents scientifiques de niveau recherche, publiés ou non, émanant des établissements d'enseignement et de recherche français ou étrangers, des laboratoires publics ou privés. 


\title{
Indirect Tertiary Alcohol Enantiocontrol by Acylative Organocatalytic Kinetic Resolution
}

\author{
Titouan Desrues," Xueyang Liu," Jean-Marc Pons, Valérie Monnier, Jean-Arthur Amalian,
} Laurence Charles, Adrien Quintard,* and Cyril Bressy*

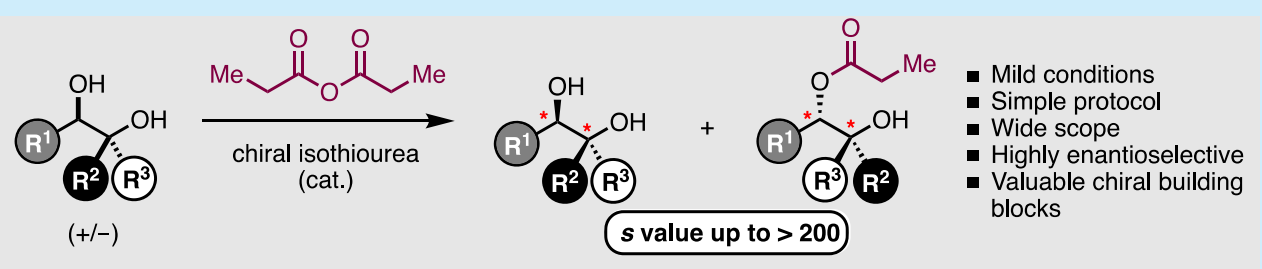

ABSTRACT: The stereocontrol of tertiary alcohols represents a recurrent challenge in organic synthesis. In the present paper, we describe a simple, efficient, and indirect method to enantioselectively prepare tertiary alcohols through a chiral isothiourea catalyzed selective acylation of adjacent secondary alcohols. This transformation enables the kinetic resolution (KR) of easily prepared racemic diastereoenriched secondary/tertiary diols providing both monoesters and starting diols in highly enantioenriched forms $(s$-value $>200$ ).

tereogenic tertiary alcohol is a ubiquitous motif present in $\checkmark$ natural products and biological active compounds (Scheme 1a). ${ }^{1}$ Chiral tertiary alcohols can also serve as building blocks to elaborate more complex molecules. The stereocontrol of this functional group remains a modern challenge as demonstrated in the Aggarwal elegant work showing a pathway between optically active secondary and tertiary alcohols. ${ }^{2}$ Enantioselective catalytic methods available to obtain tertiary alcohols (Scheme $1 \mathrm{~b}$ ) are confronted to several difficulties. Indeed, the addition of an organometallic species to a ketone, ${ }^{1}$ one of the most direct methods, suffers from severe drawbacks. While it is most simple with aldehydes, the addition on less reactive ketones additionally requires to distinguish between two potentially similar $\mathrm{R}^{1}$ and $\mathrm{R}^{2}$ groups. Various alternatives such as enantioselective dihydroxylation, ${ }^{3}$ kinetic resolution (KR) of racemic epoxides, ${ }^{4}$ desymmetrization of prochiral tertiary alcohols, ${ }^{5}$ or enantioselective $\alpha$ hydroxylation of carbonyl compounds ${ }^{6}$ have been developed. However, once again, they all require an efficient discrimination between two to three potentially structurally similar substituents.

An alternative to prepare enantioenriched tertiary alcohols is to start from easily prepared racemic tertiary alcohols. However, the first nonenzymatic catalytic $\mathrm{KR}^{7}$ of tertiary alcohols ${ }^{8,9}$ were only recently investigated. A limited number of studies was reported mainly through direct strategies, i.e., by modification of the alcohol function. Once again, the difficulty to control the chiral environment around the poorly reactive alcohol increases the challenge associated with such an approach (Scheme 2a).

Several KR are based on a stereoablative approach, ${ }^{10}$ i.e., on the destruction of the stereocenter of one enantiomer of the tertiary alcohol by breaking a C-C bond. ${ }^{8 \mathrm{a}-\mathrm{c}, \mathrm{e}, \mathrm{k}}$ Activation of racemic tertiary alcohols by chiral phosphoric acids enabled enantioselective intra- and intermolecular acetalization ${ }^{8 \mathrm{f}-\mathrm{h}}$ or heterocyclization..$^{8 \mathrm{i}, \mathrm{m}, \mathrm{n}}$ Recently an enantioselective coppercatalyzed silylation of tertiary alcohols was described forming optically active silylethers. ${ }^{81}$ Avoiding the challenging reactions directly on hindered tertiary alcohols, indirect control by reacting on an adjacent functionality has been developed (Scheme 2a). In this context, Hoveyda and Snapper showed, on three examples, the possibility to perform an enantioselective silylation of a racemic mixture of primary/tertiary diols. ${ }^{8 \mathrm{~d}}$ Yamamoto demonstrated, with two examples, the possibility to kinetically resolve tertiary allylic alcohols by enantioselective epoxidation of adjacent alkenes. ${ }^{8 g}$ Recently Hashimoto and Maruoka described an enantioselective alkylation of a racemic mixture of primary/tertiary diols occurring on the primary alcohol. ${ }^{8 j}$ In all these examples, an 
Scheme 1. (a) Natural Products Containing Stereogenic Tertiary Alcohols; (b) Main Catalytic Enantioselective Preparation of Tertiary Alcohols
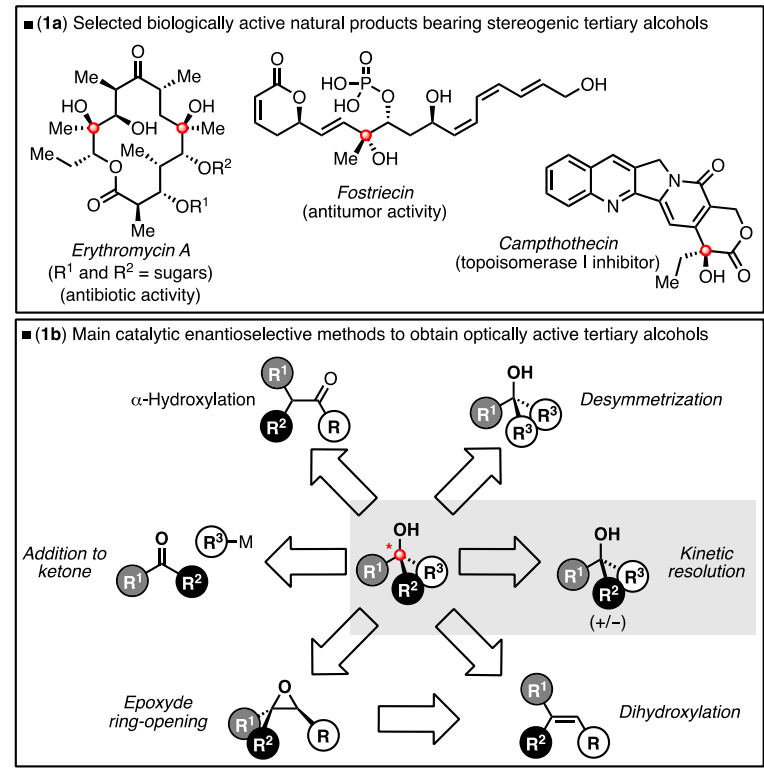

Scheme 2. Direct and Indirect Enantiocontrol of Tertiary Alcohols by Acylative Catalytic KR

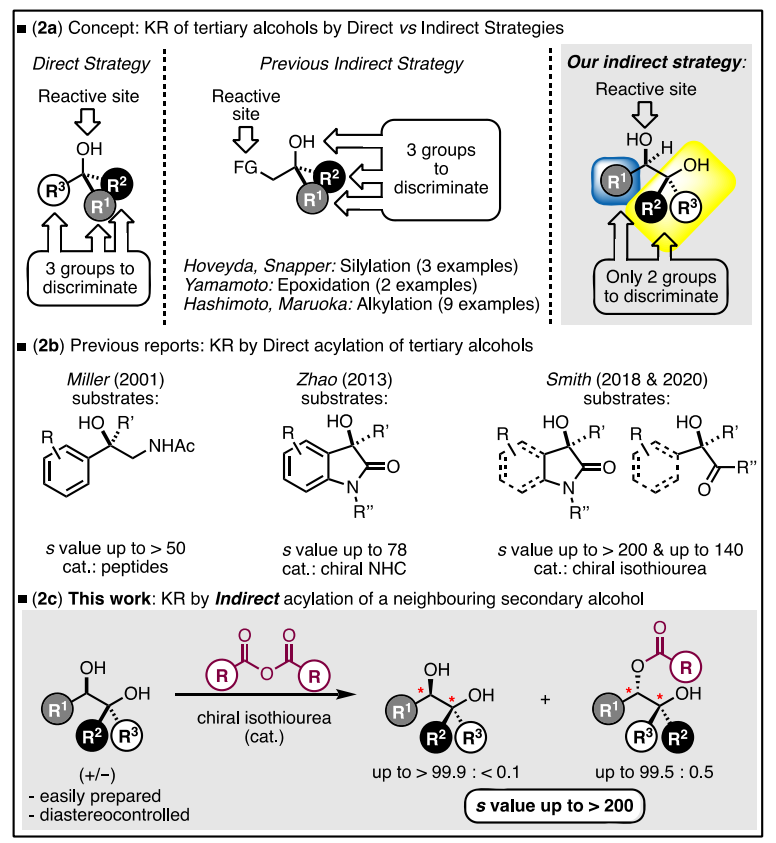

efficient discrimination between three substituents, required to obtain the enantioenriched tertiary alcohols, considerably limits the scope (Scheme 2a). In order to tackle this challenge, an indirect control through $\mathrm{KR}$ of a chiral adjacent functionality could represent a valuable alternative.

While the enantioselective acylation of secondary alcohols is quite well established, as for the other above-mentioned KR, the acylation of tertiary ones is more challenging. Using enantioselective organocatalyzed processes, only three reports of direct acylation bypass the inherent limited reactivity of such alcohols (Scheme $2 b$ ). Miller ${ }^{9 a}$ used oligopeptides to perform the enantioselective acylation of tertiary alcohols bearing an acetamide functionality as recognition group. Zhao ${ }^{9 \mathrm{~b}}$ and Smith ${ }^{9 \mathrm{c}}$ used respectively chiral NHC and chiral isothiourea to catalyze the acylation of isatin derivatives. The Smith's study revealed a privileged interaction between the adjacent $\mathrm{C}=\mathrm{O}$ and the acylated catalyst leading to an efficient discrimination between the three substituents. In addition, in 2020, Smith explored acyclic substrates with $s$-values up to $140 .^{9 \mathrm{~d}}$

In this context, we hypothesized that kinetic resolution of a chiral secondary alcohol could control a beforehand diastereoselectively easily generated adjacent tertiary alcohol (Scheme $2 a$ and $2 c$ ). These secondary/tertiary diols are easily accessible through direct diastereoselective nucleophilic addition on the corresponding ketones and such structure is present in numerous natural products (see erythromycin A and fostriecin in Scheme 1a). This strategy would take advantage of the excellent enantiocontrol observed in the KR of a variety of diversely functionalized secondary alcohols, i.e., the enantiodiscrimination between two groups. The presence of the tertiary alcohol should provide a huge steric hindrance close to the reactive center leading to an improved discrimination between the substituents of the secondary alcohol resulting in excellent selectivity, i.e., with selectivity factor $(s)$ above 20 . In this manuscript, we report our findings on this KR indirect process to isolate tertiary alcohols with almost perfect enantiocontrol. In this process, the enantiodiscriminating event takes place only on the secondary alcohol and leads to a broad scope of synthetically relevant products.

A first screening of commercially available isothiourea catalysts $^{11}$ was performed at room temperature on diastereopure diol 1a (Table 1, entries 1-4). In contrast with our

Table 1. Optimization of the KR
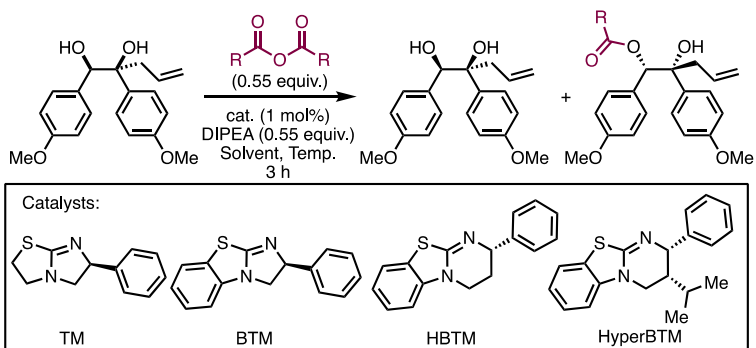

\begin{tabular}{crllrrr} 
entry & $\mathrm{R}$ & \multicolumn{1}{c}{ solvent } & catalyst & $\begin{array}{r}\text { temp } \\
\left({ }^{\circ} \mathrm{C}\right)\end{array}$ & $\begin{array}{r}\mathrm{C}^{a} \\
(\%)\end{array}$ & \multicolumn{1}{c}{$s^{a}$} \\
1 & $\mathrm{Et}$ & $\mathrm{CH}_{2} \mathrm{Cl}_{2}$ & TM & 20 & 50 & 31 \\
2 & $\mathrm{Et}$ & $\mathrm{CH}_{2} \mathrm{Cl}_{2}$ & BTM & 20 & 49 & 110 \\
3 & $\mathrm{Et}$ & $\mathrm{CH}_{2} \mathrm{Cl}_{2}$ & HBTM & 20 & 48 & 32 \\
4 & $\mathrm{Et}$ & $\mathrm{CH}_{2} \mathrm{Cl}_{2}$ & HyperBTM & 20 & 49 & 180 \\
5 & $\mathrm{Et}$ & $\mathrm{CH}_{2} \mathrm{Cl}_{2}$ & HyperBTM & 0 & 49 & 170 \\
6 & $E t$ & $\mathrm{CH}_{2} \mathrm{Cl}_{2}$ & HyperBTM & -20 & 49 & $>200$ \\
7 & $\mathrm{Et}$ & $\mathrm{MeOCO}_{2} \mathrm{Me}$ & HyperBTM & +6 & 48 & 140 \\
8 & $\mathrm{Et}$ & $t-\mathrm{AmOH}_{2}$ & HyperBTM & -10 & 34 & 10 \\
9 & $\mathrm{Me}$ & $\mathrm{CH}_{2} \mathrm{Cl}_{2}$ & HyperBTM & -20 & 52 & 140 \\
10 & $i-\mathrm{Pr}$ & $\mathrm{CH}_{2} \mathrm{Cl}_{2}$ & HyperBTM & -20 & 21 & $>200$
\end{tabular}

${ }^{a}$ Determined using Kagan's equations: $C=\mathrm{ee}_{\text {substrate }} /\left(\mathrm{ee}_{\text {substrate }}+\right.$ ee $\left.e_{\text {product }}\right)$ and $s=\left(\ln \left(1-C\left(1+e_{\text {product }}\right)\right)\right) /(\ln (1-C(1-$ $\left.\left.\left(e_{\text {product }}\right)\right)\right)$. 
previous studies on acylation of diols where a double acylation occurs leading to an amplification of enantioselectivity, ${ }^{12}$ a single acylation occurred on the secondary hydroxyl group with high level of selectivity, while no tertiary alcohol acylation was observed. However, according to our hypothesis, this single acylation event was sufficient to provide excellent selectivity. Tetramisole (TM) (entry 1), benzotetramisole $(\text { BTM })^{13}$ (entry 2), and homotetramisole (HBTM) ${ }^{14}$ (entry 3 ) gave promising results with respectively $s=31, s=110$, and $s=32$ using propionic anhydride as the acylating reagent. Rigidifying the catalyst structure in HyperBTM ${ }^{15}$ enabled an increase of the $s$-value up to 179 (entry 4). When decreasing the temperature to $-20{ }^{\circ} \mathrm{C}$, with the same catalyst, a higher $s$ value $>200$ was obtained (entry 6). Solvents screening did not improve the $s$-values (entries 7 and 8). Smaller acylating reagent decreased the selectivity (entry 9), while use of the bulkier isobutyric anhydride provided an excellent $s$-value at the expense of a low conversion (entry 10). These results prompted us to keep the more reactive propionic anhydride and conditions depicted in entry 6 as standards.

With optimized conditions in hand, we explored the scope of this catalytic KR (Scheme 3). The influence of the nature of

\section{Scheme 3. Scope of the KR}

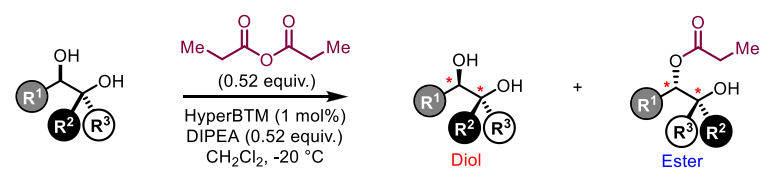

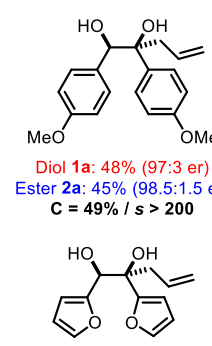

Diol 1d: $45 \%(91: 9 \mathrm{er})$ Ester 2d: $48 \%(91: 9 \mathrm{er})$
$\mathbf{C}=\mathbf{5 0} \% / \mathrm{s}=\mathbf{2 6}$

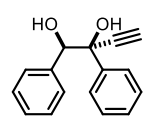

Diol 1g: $36 \%$ (94.5:5.5 er) Ester 2g: $37 \%(93.5: 6.5 \mathrm{er})$
$\mathbf{C}=\mathbf{4 9 \%} / \mathbf{s}=\mathbf{4 3}$

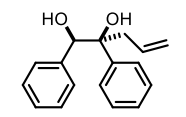

Diol $1 \mathrm{~b}: 40 \%(99.5: 0.5 \mathrm{er})$ Ester $\mathbf{2 b}: \mathbf{3 7 \%}(98: 2$ er)
$\mathbf{C}=\mathbf{5 1} \% / \mathbf{s}>\mathbf{2 0 0}$

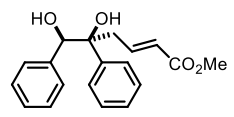

Diol 1e: $45 \%$ (>99.9:0.1 er) (RX) Ester $2 \mathrm{e}: 46 \%(98.5: 1.5 \mathrm{er})$
$\quad \mathbf{C}=\mathbf{5 1 \%} / \mathrm{s}>\mathbf{2 0 0}$

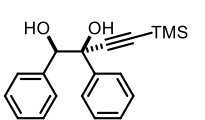

Diol $1 \mathrm{~h}: 44 \%$ (93.5:6.5 er) Ester $\mathbf{2 h}: 36 \%(96.5: 3.5 \mathrm{er})$
$\mathbf{C}=\mathbf{4 8 \%} / \boldsymbol{s}=\mathbf{7 0}$

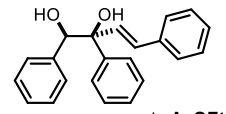

in AcOEt, $-20^{\circ} \mathrm{C}$ Diol 1j: 36\% (62.5:37.5 er) \begin{tabular}{l|l} 
Diol 1j: $36 \%(72.2: 27.8$ er \\
(n)
\end{tabular}

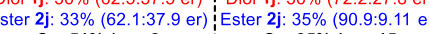

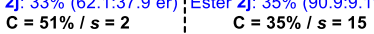

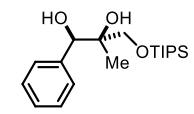

Diol 11: $54 \%(79: 21 \mathrm{er})$ $\mathbf{C}=\mathbf{4 0} \% / \mathbf{s}=\mathbf{2 4}$
$\mathbf{C}$ the aromatic substituents was investigated first. Comparable results were obtained with a diol bearing two phenyl groups such as diol $\mathbf{1 b}$. The highest selectivity was observed with diol 1c presenting two different aryl groups reaching a $s$-value $>200$ meaning that the recovered diol and the produced monoester are obtained nearly enantiopure at $50 \%$ conversion. A drop of selectivity was observed with diol $\mathbf{1} \mathbf{d}$, bearing two furan groups, probably due to a lower recognition of the furan by the catalyst. In contrast excellent results were achieved by functionalizing the allylic side chain as shown with diols 1e and 1f. X-ray diffraction analysis of crystals of enantiopure diol 1e confirmed the absolute stereochemistry. ${ }^{16}$ It is in accordance with the stereochemical model of the transition state where the aromatic substituent linked to the secondary alcohol is the recognizing motif interacting by $\pi-\pi$ or cation $-\pi$ interaction.

The KR of several diols (1g, 1h, 1i), with a propargylic tertiary alcohol, was then investigated (Scheme 3). Surprisingly, the nature of the alkyne substituent influenced significantly the KR outcome. Indeed, terminal alkyne $\mathbf{1 g}$ gave an $s$-value of 43 . Substitution with a trimethylsilyl group (1h) improved the selectivity to $s=70$, while substitution with a phenyl group (1i) reduced the selectivity to $s=6$. We also explored different hybridizations $\left(\mathrm{sp}, \mathrm{sp}^{2}, \mathrm{sp}^{3}\right)$ of the tertiary alcohol substituents in substrates $\mathbf{1 i}, \mathbf{1 j}$, and $\mathbf{1 k}$. The presence of the styrenyl group in diol $\mathbf{1} \mathbf{j}$ is deleterious to the selectivity, while a $\mathrm{sp}^{3}$ substituent improves the results as shown with diol 1k. Collectively, these results suggest that competing $\pi$ interactions can decrease the selectivity if an extended conjugated system is adjacent to the tertiary alcohol. Moving to AcOEt as solvent limits these undesired interactions as demonstrated in the KR of diol $\mathbf{1 j}$ where an increased $s$-value of 15 is observed. Finally, the KR of the two diastereomers 11 and $\mathbf{1 m}$ was examined. Gratifyingly, both diastereomers provided a good selectivity factor above 20 , confirming the largely predominant role of the secondary alcohol configuration on the stereochemical outcome. Observation of a higher selectivity for one of the two diastereomers also suggests that secondary interactions possibly with the free alcohol might slightly modulate the catalyst control.

To demonstrate the applicability of our method, we also explored the scalability of this transformation on the gram scale with the racemic diol 1c (Scheme 4). Under the same

Scheme 4. Scalability of the KR

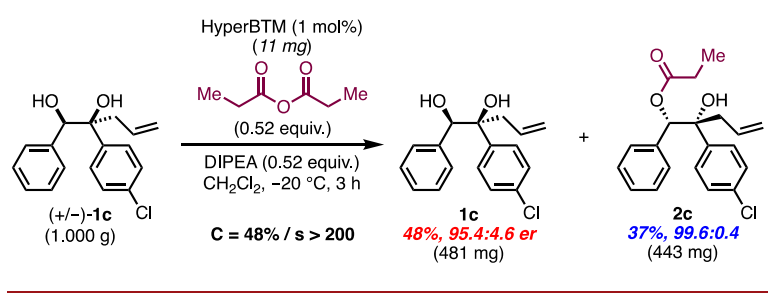

conditions, similar results were obtained on this preparative scale using only $11 \mathrm{mg}$ of HyperBTM (1 mol \%). Indeed, enantioenriched diol 1c was recovered in $48 \%$ yield $(481 \mathrm{mg}$ ) with an er $=95.4: 4.6$ and the corresponding monoester was obtained in $37 \%$ yield $(443 \mathrm{mg}$ ) with an er $=99.6: 0.4$.

To explore the limitation of this methodology, secondary/ tertiary 1,3-diol 1 n was also tested under the optimized enantioselective acylation conditions. Interestingly, despite the remoteness of the tertiary alcohol decreasing the favorable 
steric interactions in the enantiodiscriminating event, an $s$ value of 11 was still observed with this challenging substrate (Scheme 5).

\section{Scheme 5. KR of 1,3-Diol 1n}

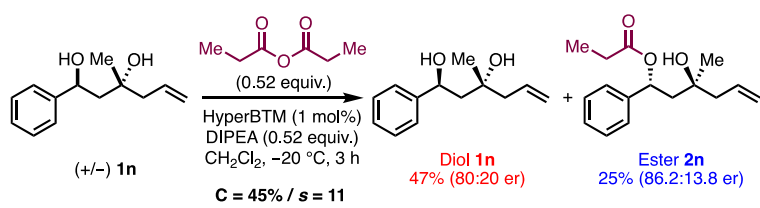

With this methodology in hand, we explored the synthetic potential of the highly enantioenriched building block 1c bearing a tertiary alcohol (Scheme 6). Secondary alcohol

Scheme 6. Postfunctionalizations of Diol 1c

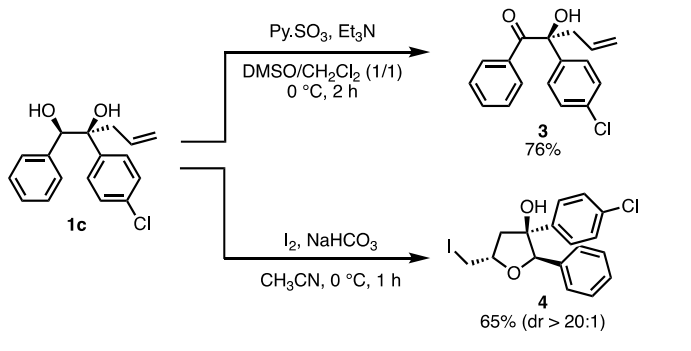

oxidation using pyridine- $\mathrm{SO}_{3}$ complex in the presence of triethylamine afforded hydroxyketone 3 , possessing exclusively a tertiary alcohol, in $76 \%$ yield. ${ }^{17}$ This result demonstrates the power of our approach to control the configuration of such challenging tertiary alcohols in a traceless manner. Treatment of diol 1c with molecular iodine in acetonitrile led to the diastereoselective formation $(\mathrm{dr}>20: 1)$ of the highly substituted tetrahydrofuran 4 in $65 \%$ yield.

In conclusion, we described a synthetically useful method to perform a catalytic kinetic resolution of secondary/tertiary 1,2diols. It constitutes an indirect acylative alternative to control the stereochemistry of tertiary alcohols known as a relevant synthetic problem. This simple and scalable protocol showed high $s$-values providing highly enantioenriched valuable chiral building blocks.

\section{Accession Codes}

CCDC 2012308 and 2012311 contain the supplementary crystallographic data for this paper. These data can be obtained free of charge via www.ccdc.cam.ac.uk/data_request/cif, or by emailing data_request@ccdc.cam.ac.uk, or by contacting The Cambridge Crystallographic Data Centre, 12 Union Road, Cambridge CB2 1EZ, UK; fax: +44 1223336033.

\section{AUTHOR INFORMATION}

\section{Corresponding Authors}

Adrien Quintard - Aix Marseille Univ, CNRS, Centrale Marseille, iSm2, Marseille, France; @ orcid.org/0000-00030193-6524; Email: adrien.quintard@univ-amu.fr

Cyril Bressy - Aix Marseille Univ, CNRS, Centrale Marseille, iSm2, Marseille, France; ๑orcid.org/0000-0003-29448274; Email: cyril.bressy@univ-amu.fr

\section{Authors}

Titouan Desrues - Aix Marseille Univ, CNRS, Centrale Marseille, iSm2, Marseille, France

Xueyang Liu - Aix Marseille Univ, CNRS, Centrale Marseille, iSm2, Marseille, France

Jean-Marc Pons - Aix Marseille Univ, CNRS, Centrale Marseille, iSm2, Marseille, France

Valérie Monnier - Aix Marseille Univ, CNRS, Centrale Marseille, FSCM, Marseille, France

Jean-Arthur Amalian - Aix Marseille Univ, CNRS, ICR, Marseille, France

Laurence Charles - Aix Marseille Univ, CNRS, ICR, Marseille, France; ๑ orcid.org/0000-0003-3807-8375

\section{Author Contributions \\ ${ }$ T.D. and X.L. contributed equally to this work. Notes}

The authors declare no competing financial interest.

\section{ACKNOWLEDGMENTS}

We warmly thank personnel from Aix Marseille University, Dr. N. Vanthuyne, M. Jean (HPLC), and Dr. M. Giorgi (X-ray diffraction). Aix Marseille University, CNRS, and ANR (Agence Nationale de la Recherche) (AMPLI project ANR18-CE07-0036) are gratefully acknowledged for funding.

\section{REFERENCES}

(1) For reviews, see: (a) Riant, O.; Hannedouche, J. Asymmetric catalysis for the construction of quaternary carbon centres: nucleophilic addition on ketones and ketimines. Org. Biomol. Chem. 2007, 5, 873-888. (b) Shibasaki, M.; Kanai, M. Asymmetric Synthesis of Tertiary Alcohols and $\alpha$-Tertiary Amines via $\mathrm{Cu}$ Catalyzed C-C Bond Formation to Ketones and Ketimines. Chem. Rev. 2008, 108, 2853-2873. (c) Liu, Y. L.; Lin, X.-T. Recent Advances in Catalytic Asymmetric Synthesis of Tertiary Alcohols via Nucleophilic Addition to Ketones. Adv. Synth. Catal. 2019, 361, 876918.

(2) (a) Stymiest, J. L.; Bagutski, V.; French, R. M.; Aggarwal, V. K. Enantiodivergent conversion of chiral secondary alcohols into tertiary alcohols. Nature 2008, 456, 778-783. (b) Pulis, A. P.; Varela, A.; Citti, C.; Songara, P.; Leonori, D.; Aggarwal, V. K. Asymmetric Synthesis of Tertiary Alcohols and Thiols via Nonstabilized Tertiary $\alpha$-Oxy- and $\alpha$-Thio-Substituted Organolithium Species. Angew. Chem., Int. Ed. 2017, 56, 10835-10839.

(3) Selected example: Sharpless, K. B.; Amberg, W.; Bennani, Y. L.; Crispino, G. A.; Hartung, J.; Jeong, K. S.; Kwong, H.-L.; Morikawa, K.; Wang, Z.-M.; Xu, D.; Zhang, X. L. The osmium-catalyzed asymmetric dihydroxylation: a new ligand class and a process improvement. J. Org. Chem. 1992, 57, 2768-2771.

(4) Selected example: Lebel, H.; Jacobsen, E. N. Chromium catalyzed kinetic resolution of 2,2-disubstituted epoxides. Tetrahedron Lett. 1999, 40, 7303-7306. 
(5) Selected example: Jung, B.; Hong, M. S.; Kang, S. H. Enantioselective Synthesis of Tertiary Alcohols by the Desymmetrizing Benzoylation of 2-Substituted Glycerols. Angew. Chem., Int. Ed. 2007, 46, 2616-2618.

(6) Selected example: Yang, F.; Zhao, J.; Tang, X.; Zhou, G.; Song, W.; Meng, Q. Enantioselective $\alpha$-Hydroxylation by Modified SalenZirconium(IV)-Catalyzed Oxidation of $\beta$-Keto Esters. Org. Lett. 2017, $19,448-451$.

(7) (a) Kagan, H. B.; Fiaud, J.-C. Kinetic Resolution. Top. Stereochem. 1988, 18, 249-330. (b) Keith, M. J.; Larrow, J. F.; Jacobsen, E. N. Practical Considerations in Kinetic Resolution Reactions. Adv. Synth. Catal. 2001, 343, 5-26. (c) Vedejs, E.; Jure, M. Efficiency in Nonenzymatic Kinetic Resolution. Angew. Chem., Int. Ed. 2005, 44, 3974-4001. (d) Müller, C. E.; Schreiner, P. R. Organocatalytic Enantioselective Acyl Transfer onto Racemic as well as meso Alcohols, Amines, and Thiols. Angew. Chem., Int. Ed. 2011, 50, 6012-6042. (e) Gurubrahamam, R.; Cheng, Y.-S.; Huang, W.-Y.; Chen, K. Recent Advances in Organocatalytic Kinetic Resolution for the Synthesis of Functionalized Products. ChemCatChem 2016, 8, 86-96. (f) Greenhalgh, M. D.; Taylor, J. E.; Smith, A. D. Best practice considerations for using the selectivity factor, $s$, as a metric for the efficiency of kinetic resolutions. Tetrahedron 2018, 74, 5554-5560.

(8) (a) Wang, F.; Tu, Y. Q.; Fan, C. A.; Wang, S. H.; Zhang, F. M. Kinetic resolution via semipinacol rearrangement of $\alpha$-hydroxy epoxides: a new method for asymmetric synthesis of $\alpha$-hydroxy epoxides and $\beta$-hydroxy ketones containing an $\alpha$-quaternary carbon. Tetrahedron: Asymmetry 2002, 13, 395-398. (b) Nishimura, T.; Matsumura, S.; Maeda, Y.; Uemura, S. Palladium-catalyzed kinetic resolution of tert-cyclobutanols via $\mathrm{C}-\mathrm{C}$ bond cleavage. Tetrahedron Lett. 2002, 43, 3037-3039. (c) Tosaki, S.-Y.; Hara, K.; Gnanadesikan, V.; Morimoto, H.; Harada, S.; Sugita, M.; Yamagiwa, N.; Matsunaga, S.; Shibasaki, M. Mixed La-Li Heterobimetallic Complexes for Tertiary Nitroaldol Resolution. J. Am. Chem. Soc. 2006, 128, 1177611777. (d) Zhao, Y.; Mitra, A. W.; Hoveyda, A. H.; Snapper, M. Kinetic Resolution of 1,2-Diols through Highly Site- and Enantioselective Catalytic Silylation. Angew. Chem., Int. Ed. 2007, 46, 84718474. (e) Shintani, R.; Takatsu, K.; Hayashi, T. Rhodium-Catalyzed Kinetic Resolution of Tertiary Homoallyl Alcohols via Stereoselective Carbon-Carbon Bond Cleavage. Org. Lett. 2008, 10, 1191-1193.

(f) Coric, I.; Müller, S.; List, B. Kinetic Resolution of Homoaldols via Catalytic Asymmetric Transacetalization. J. Am. Chem. Soc. 2010, 132 (49), 17370-17373. (g) Olivares-Romero, J. L.; Li, Z.; Yamamoto, H. Catalytic Enantioselective Epoxidation of Tertiary Allylic and Homoallylic Alcohols. J. Am. Chem. Soc. 2013, 135, 3411-3413.

(h) Kim, J. H.; Coric, I.; Palumbo, C.; List, B. Resolution of Diols via Catalytic Asymmetric Acetalization. J. Am. Chem. Soc. 2015, 137, 1778-1781. (i) Yoneda, N.; Matsumoto, A.; Asano, K.; Matsubara, S. Asymmetric Cycloetherification via the Kinetic Resolution of Alcohols Using Chiral Phosphoric Acid Catalysts. Chem. Lett. 2016, 45, 13001303. (j) Pawliczek, M.; Hashimoto, T.; Maruoka, K. Alkylative kinetic resolution of vicinal diols under phase-transfer conditions: a chiral ammonium borinate catalysis. Chem. Sci. 2018, 9, 1231-1235. (k) Zhang, W.; Ma, S. Palladium/H+-cocatalyzed kinetic resolution of tertiary propargylic alcohols. Chem. Commun. 2018, 54, 6064-6067. (1) Seliger, J.; Dong, X.; Oestreich, M. Kinetic Resolution of Tertiary Propargylic Alcohols by Enantioselective $\mathrm{Cu}-\mathrm{H}-$ Catalyzed $\mathrm{Si}-\mathrm{O}$ Coupling. Angew. Chem., Int. Ed. 2019, 58, 1970-1974. (m) Rajkumar, S.; He, S.; Yang, X. Kinetic Resolution of Tertiary 2-Alkoxycarboxamido-Substituted Allylic Alcohols by Chiral Phosphoric Acid Catalyzed Intramolecular Transesterification. Angew. Chem., Int. Ed. 2019, 58, 10315-10319. (n) Rajkumar, S.; Tang, M.; Yang, X. Chiral Phosphoric Acid Catalyzed Kinetic Resolution of 2-Amido Benzyl Alcohols: Asymmetric Synthesis of 4H-3,1-Benzoxazines. Angew. Chem., Int. Ed. 2020, 59, 2333-2337.

(9) (a) Jarvo, E. R.; Evans, C. A.; Copeland, G. T.; Miller, S. J. Fluorescence-Based Screening of Asymmetric Acylation Catalysts through Parallel Enantiomer Analysis. Identification of a Catalyst for Tertiary Alcohol Resolution. J. Org. Chem. 2001, 66, 5522-5527. (b) Lu. S.: Poh. S. B.; Siau. W.-Y.; Zhao, Y. Kinetic Resolution of
Tertiary Alcohols: Highly Enantioselective Access to 3-Hydroxy-3Substituted Oxindoles. Angew. Chem., Int. Ed. 2013, 52, 1731-1734. (c) Greenhalgh, M. D.; Smith, S. M.; Walden, D. M.; Taylor, J. E.; Brice, Z.; Robinson, E. R. T.; Fallan, C.; Cordes, D. B.; Slawin, A. M. Z.; Richardson, H. C.; Grove, M. A.; Cheong, P. H-Y.; Smith, A. D. A $\mathrm{C}=\mathrm{O} \cdots$ Isothiouronium Interaction Dictates Enantiodiscrimination in Acylative Kinetic Resolutions of Tertiary Heterocyclic Alcohols. Angew. Chem., Int. Ed. 2018, 57, 3200-3206. (d) Qu, S.; Smith, S. M.; Laina-Martin, V.; Neyyappadath, R. M.; Greenhalgh, M. D.; Smith, A. D. Angew. Chem., Int. Ed. 2020, 59, 16572-16578.

(10) For a review, see: Mohr, J. T.; Ebner, D. C.; Stoltz, B. M. Catalytic enantioselective stereoablative reactions: an unexploited approach to enantioselective catalysis. Org. Biomol. Chem. 2007, 5, 3571-3576.

(11) For reviews, see: (a) Merad, J.; Pons, J.-M.; Chuzel, O.; Bressy, C. Enantioselective Catalysis by Chiral Isothioureas. Eur. J. Org. Chem. 2016, 2016, 5589-5610. (b) Birman, V. B. Amidine-Based Catalysts (ABCs): Design, Development, and Applications. Aldrichimica Acta 2016, 49, 23-33.

(12) (a) Merad, J.; Borkar, P.; Caijo, F.; Pons, J.-M.; Parrain, J.-L.; Chuzel, O.; Bressy, C. Double Catalytic Kinetic Resolution (DoCKR) of Acyclic anti-1,3-Diols: The Additive Horeau Amplification. Angew. Chem., Int. Ed. 2017, 56, 16052-16056. (b) Merad, J.; Borkar, P.; Bouyon Yenda, T.; Roux, C.; Pons, J.-M.; Parrain, J.-L.; Chuzel, O.; Bressy, C. Highly Enantioselective Acylation of Acyclic Meso 1,3Diols through Synergistic Isothiourea-Catalyzed Desymmetrization/ Chiroablative Kinetic Resolution. Org. Lett. 2015, 17, 2118-2121. (c) Harrer, S.; Greenhalgh, M. D.; Neyyappadath, R. M.; Smith, A. D. Isothiourea-Catalysed Sequential Kinetic Resolution of Acyclic (+)-1,2-Diols. Synlett 2019, 30, 1555-1560. For a review, see: (d) Merad, J.; Candy, M.; Pons, J.-M.; Bressy, C. Catalytic Enantioselective Desymmetrization of Meso Compounds in Total Synthesis of Natural Products: Towards an Economy of Chiral Reagents. Synthesis 2017, 49, 1938-1954.

(13) Birman, V. B.; Li, X. Benzotetramisole: A Remarkably Enantioselective Acyl Transfer Catalyst. Org. Lett. 2006, 8, 13511354.

(14) Zhang, Y.; Birman, V. B. Effects of Methyl Substituents on the Activity and Enantioselectivity of Homobenzotetramisole-Based Catalysts in the Kinetic Resolution of Alcohols. Adv. Synth. Catal. 2009, 351, 2525-2529.

(15) Joannesse, C.; Johnston, C. P.; Concellon, C.; Simal, C.; Philp, D.; Smith, A. D. Isothiourea-Catalyzed Enantioselective Carboxy Group Transfer. Angew. Chem., Int. Ed. 2009, 48, 8914-8918.

(16) CCDC 2012311 (isobutyrate) and CCDC 2012308 (optically active diol 1e) contain the supplementary crystallographic data available at the Cambridge Crystallographic Data Centre via www. ccdc.cam.ac.uk/data request/cif.

(17) Parikh, J. R.; Doering, W. v. E. Sulfur trioxide in the oxidation of alcohols by dimethyl sulfoxide. J. Am. Chem. Soc. 1967, 89, 55055507. 